\title{
CHROMOSPHERIC VARIATIONS \\ IN MAIN SEQUENCE STARS
}

\author{
O. C. WILSON \\ Hale Observatories, Carnegie Institution of Washington, \\ California Institute of Technology
}

The work described here is still in progress; hence this paper will be a brief sampling of the results to date.

There are several observable indices of the more or less periodic variations in solar activity known generally as the solar cycle. It has long been known that one of these indices is the total flux in the chromospheric emission components of the $\mathrm{H}$ and $\mathrm{K}$ lines of $\mathrm{Ca}$ II. In searching for analogous variations in stars, this same quantity appears to be the most easily observable one, provided suitable instrumentation is available.

About ten years ago a coude scanner was completed for the 100-in. telescope on Mt. Wilson. This is a two channel machine, with two photomultipliers and two sets of pulse counting electronics. It is arranged so that one channel receives the signal from a slit whose width can be chosen, and across which the spectrum can be moved in the direction of dispersion. The other channel receives the combined signals from two $25 \AA$ windows on either side of the region through which the spectrum can be scanned. In the present work the scan mode was not used. Taking into account the total radial velocity of a star at a given time, the second slit can be set at the centers of the $\mathrm{H}$ and $\mathrm{K}$ lines with a precision of two or three hundredths of an ångström. Both the entrance and exit slits were set for one Ångström width at the focal plane.

An observation consists of four integrations, two in $\mathrm{K}$ and two in $\mathrm{H}$, with the counts in both channels started by pushing a button and terminated simultaneously by the machine at a pre-set value of the count in the one Ångström channel. For most stars under normal conditions the latter is chosen to be $10^{4}$. The counts at the line centers are added and divided by the corresponding counts in the monitor channels, and these numbers are then divided by the same ratios obtained from a standard lamp observed each day. In this way the effect of drifts and changes in the electronics are eliminated. Standard stars are also observed each night. These are stars with minimal H-K fluxes which probably have little or no chromospheric emission and their constancy bears out this assumption.

All the stars in the program are main sequence objects. They extend from above the Sun, at spectral types about F5, down to M0. Each star is observed on every night when it is available, and every effort is made to obtain a dozen or more observations per season. This is essential since many of the stars appear to have fluctuations on a variety of time scales and as many observations as possible are necessary to give a good representation of the longer term variations.

Figure 1 shows the results on one of the standard stars and the scatter here, of the order of $\pm 2 \%$, is probably due almost entirely to the unavoidable errors of observation. This star is typical of the eighteen standards around the sky, all of which have fluxes in the range 1300 to 1500 instrumental units. 


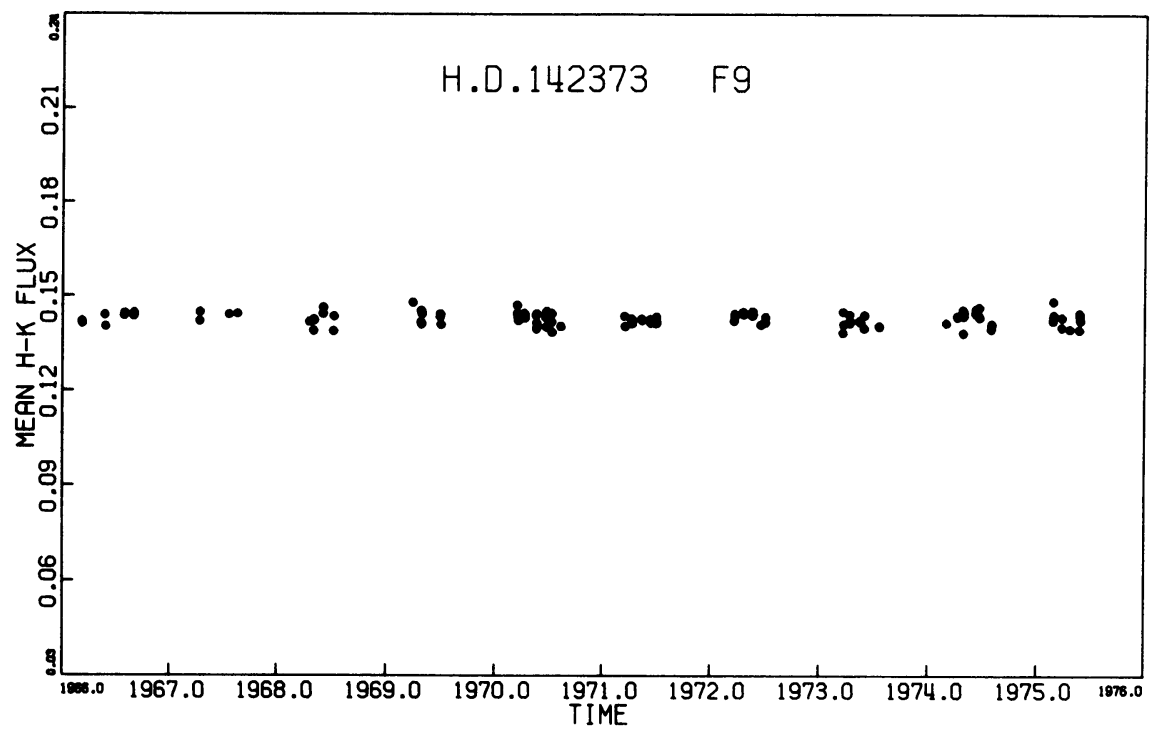

Fig. 1.

The second and third slides show the data on two stars near the Sun on the main sequence. These are typical of such objects and also of F-type stars; there are obvious variations in their fluxes, but there is no evidence for periodicity. Note that these two stars have rather strong chromospheric emissions and are therefore young. Similar stars with fluxes intermediate between the preceding ones and the standard stars, show behavior similar to that of Figures 2 and 3, but on a reduced scale.

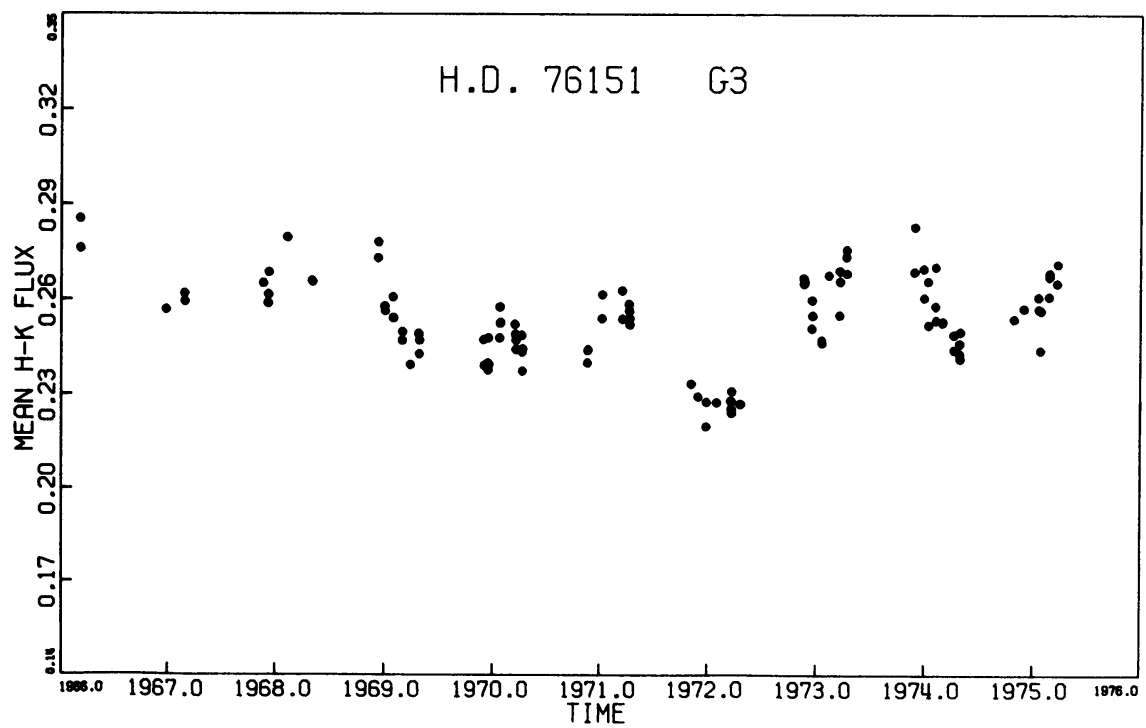

Fig. 2. 


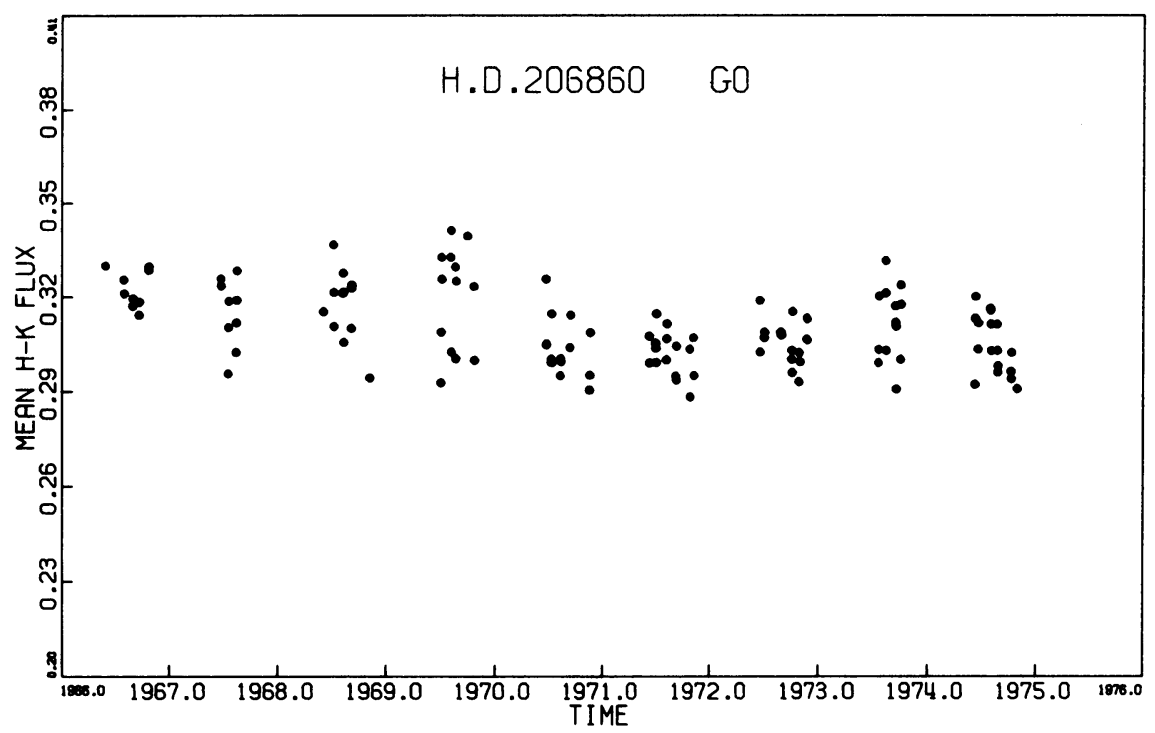

Fig. 3.

In Figures 4, 5, and 6 are shown results for three K-type stars with moderately strong emission. All three are probably undergoing cyclical variations which may well be analogous to the solar cycle. Note that the rise time seems to be shorter than the decay time in all three cases as is true for the Sun. This difference appears in exaggerated form for HD 32147 where the next rise had not yet begun even at the end of the 1974-75 observing season. The scatter in the seasonal point groups is

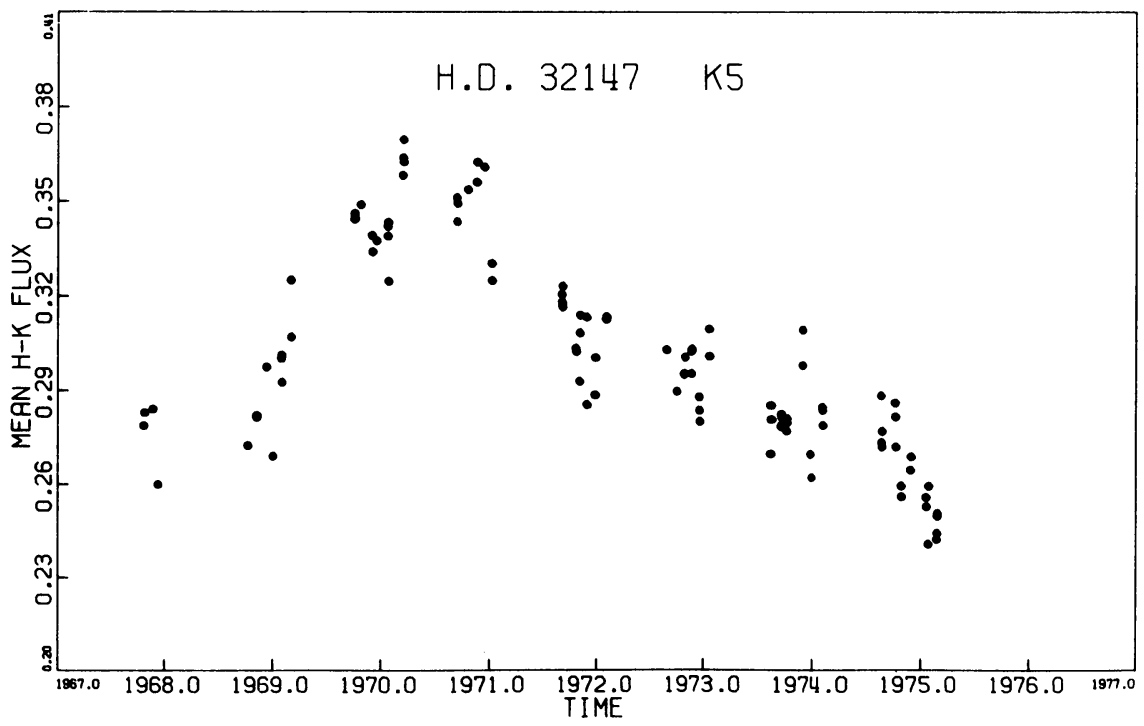

Fig. 4. 


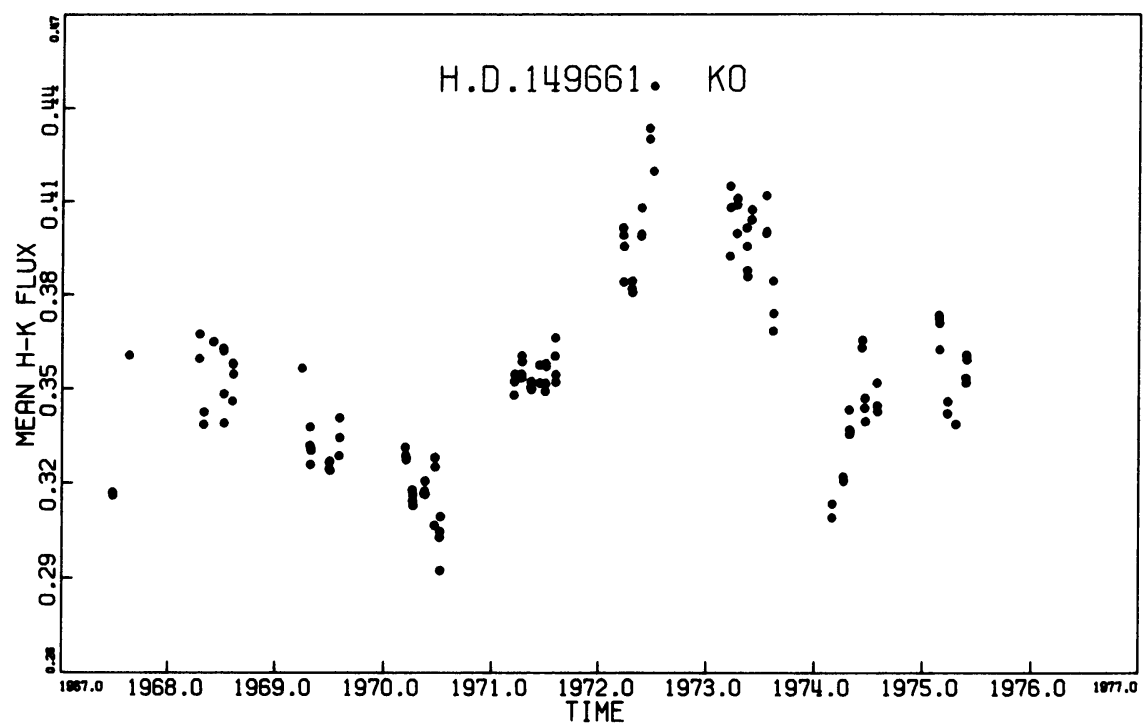

Fig. 5.

partly due to the overall change during an observing season, but a major part of it is caused by intrinsic variations of unknown source and time scale. Rotational modulation is a highly probable source for some of it, but more continuous observations would be needed to disentangle this effect from other possibly sporadic ones.

Figure 7 gives the results for another K-type star, HD 22049, which has significantly more chromospheric flux than the three preceding ones. Here there is no

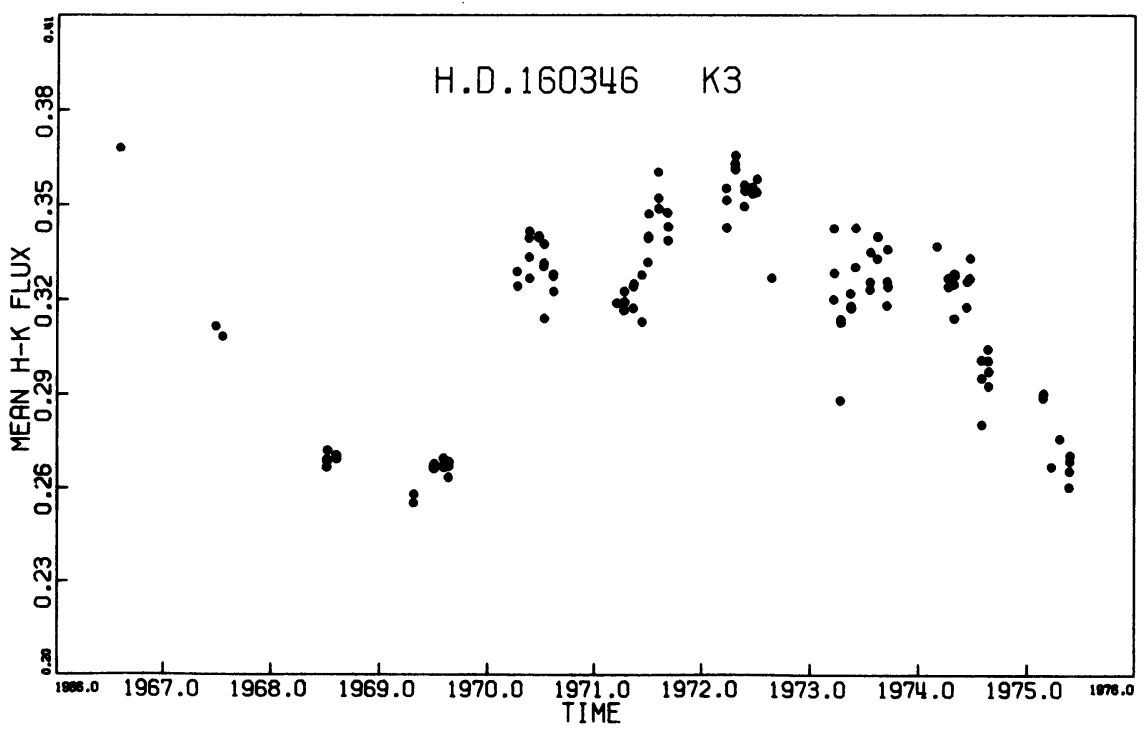

Fig. 6. 


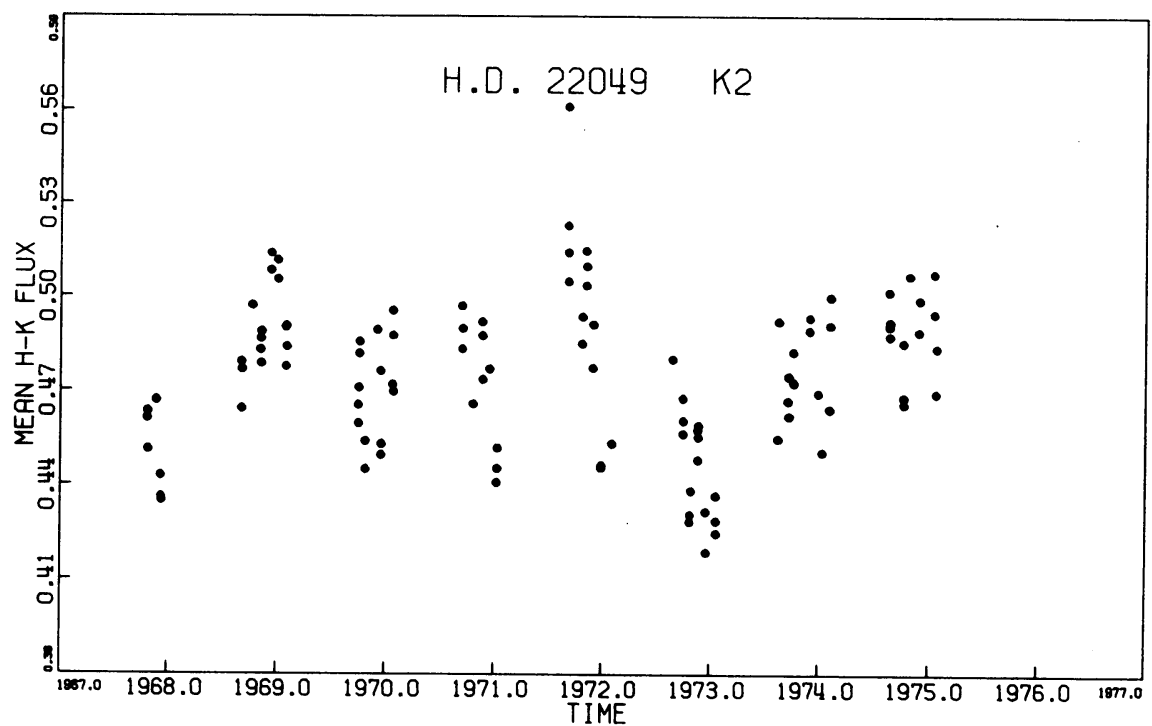

Fig. 7.

obvious cyclical variation, but there is a great deal of 'noise' or apparently random scatter. In fact, from all of the results thus far, it appears that the scatter increases with the flux.

Finally, Figure 8 shows a G8 star whose behavior is somewhat difficult to categorize, although the variations in its flux are obvious enough.

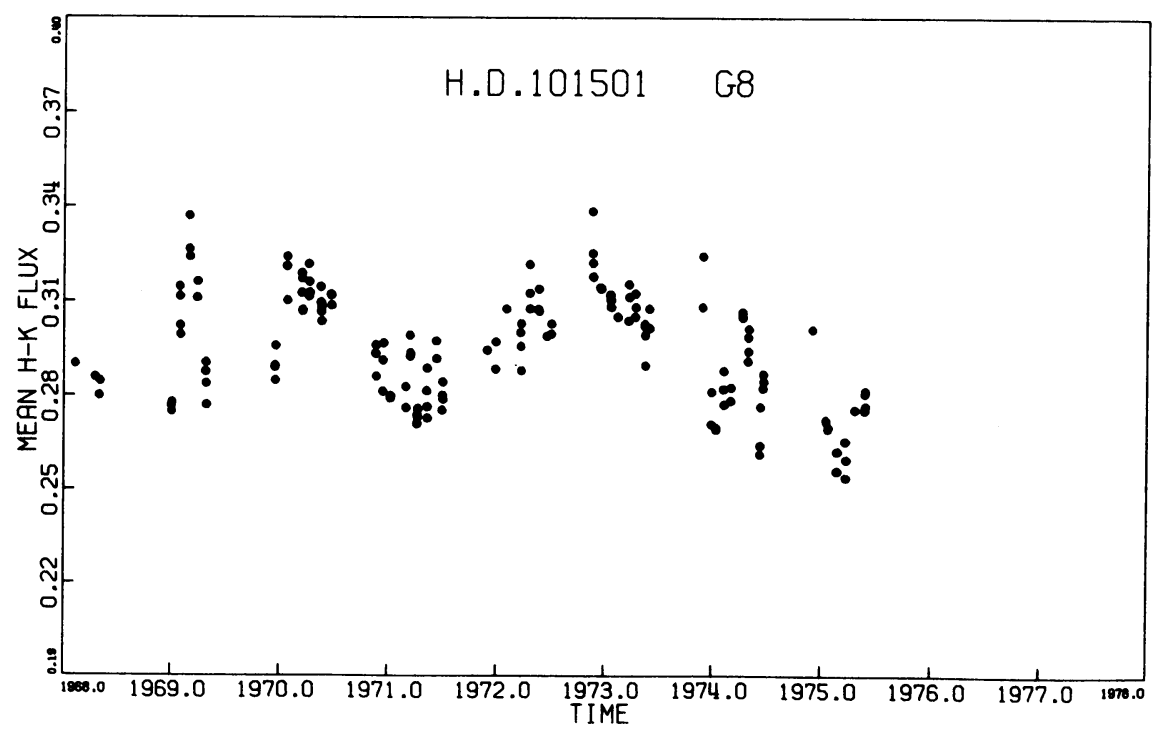

Fig. 8 . 
The samples given are a fair representation of what has been accomplished thus far. Clearly it is just a beginning, but it is hoped that observation of chromospheric variations in stars may lead to a better understanding of the solar cycle. In conclusion, it should be mentioned that none of the stars in this program are known spectroscopic binaries. 